Volume 9, No.5, September - October 2020

International Journal of Advanced Trends in Computer Science and Engineering

Available Online at http://www.warse.org/IJATCSE/static/pdf/file/ijatcse137952020.pdf

https://doi.org/10.30534/ijatcse/2020/137952020

\title{
Sustainable Object-Oriented Approach for Finding Stereographic-Projections of Hexagonal P-Groups
}

\author{
Puvvada Nagesh $^{1}$, M. Radha Madhavi ${ }^{2}$, N. Srinivasu ${ }^{3} \&$ N. Venkata Ramana ${ }^{4}$ \\ ${ }^{1,3 \& 4}$ Department of CSE, Koneru Lakshmaiah Education Foundation, Vaddeswaram, Guntur, Andhra Pradesh, \\ India-522502. PNAGESH.QA@gmail.com \\ srinivasu28@kluniversity.in \\ ramana Foundation, Vaddeswaram, Guntur, Andhra Pradesh, India-522502. \\ mrmadhavi5@gmail.com@kluniversity.in \\ ${ }^{2}$ Department of Mathematics, Koneru Lakshmaiah Education
}

\begin{abstract}
Design pattern is unique and most recent method in software-development, come into analysis from "object-oriented community". Patterns are persistent solution to several common problems. Related patterns are merged jointly forms a language to offer a method for reasonable function for software- development linked problem(s). Reasonable efforts are made and completed to join hot topic(s) by means of the transformed crystallography particularly "in the era of crystal symmetry \& physical properties". "Abstract factory technique" patterns are useful to decide physical property, demonstrated by means of the crystal belong to Hexagonal ferroic point groups (P-Groups).
\end{abstract}

Key words: Hexagonal Point Groups, Stereographic-Projections, Physical properties, Abstract Factory technique.

\section{INTRODUCTION}

The frequency of physical property(s) in crystals such-as Ferro-magnetism, density, dielectric polarization, pyro-electricity, and elasticity etc. may be calculated using symmetry-operation, linking to that crystal. The arrangement of symmetric transformations forms a special group known as the symmetric group (or) point-group. The symmetric transformations reserve the gap between pairs of point(s) for the body \& bring it in to super position with in itself. Physical property(s) of crystals can be initiated by specifying all the symmetry operation characterized by the molecular constitution or any other geometrical remains. As a result of taking into consideration the rotations, the reflection and inversion of symmetry elements there are 32 crystallographic p-groups. If the symmetry elements translation is taken into contemplation there will be total 230 space groups. A ferroic crystal contain(s) two or extra stable domain of the similar structures other than diverse spatial orientation. Aizu [2] explained all potential species of full and partial Ferro elasticity and Ferro electrics for all the 212 non-magnetic ferroic specie(s). Pree et al [ 1] worked on Design patterns of object-oriented software development Reading.Nagesh.p.et al [3] gave stereographic projections of tetragonal point groups by reusable object-oriented design. Lanka, S. et al. [4] discussed about Predictive data mining techniques of high dimensional big-data. Ramesh Babu, et.al [5] derived tensor-pairs and domain-pairs of cesium-dihydrogen phosphate using group theoretical techniques. Madhavi, R. et al.[6] solved complex engineering problems by Nature inspired techniques. Puvvada, N., [7] designed Semantic web-based banana expert system. Venkata Ramana, N., [8] Analyzed Big data and iot gadgets for tech savvy cities. Venkata Ramana, N. et al [9] Analyzed architecture to overcome real-time traffic-control as an intelligent transportation system using Big-data. Venkata Ramana, N. et al [10] Designed IoT based scientific to conquer- constant movement control as a canny transportation frame-work utilizing huge information available in cloud- networks. Rajesh, . B. et al[11] "A new scheme to safeguard data for cloud integrated internet things." Venkata Ramana, N., [12] developed Hybrid K-mir algorithm to predict type of lung cancer among stoicism. Siva Nageswara Rao.et al. [13-17] developed an Efficient PIMRR Algorithm for Improving scheduling Criteria's in Real Time Systems.

In the current paper effort has been initiated to relate the "abstract factory method" of the "Design patterns" to resolve whether a crystal exhibit a physical property(s) or not, captivating into Implement the operation(s) to carry the tasks and collaboration(s) in all the pattern(s).

\section{DESIGN PATTERNS}

Depending upon the purposes of the design pattern can be classified into three categories. 
- Creational patterns: The objects created instead of initiating them directly. Here programs gain additional flexibility in determining which object(s) need to be produced for any given scenario.

- Structured patterns: These pattern(s) help in composing group(s) of objects into bigger structure(s) such as complex interface $\&$ accounting info.

- Behavioral pattern: These patterns helps to describe communications between objects in the system \& the movement of control in a complicated program.

There are 23 design pattern(s) that were implemented in java in literature for different varieties of problems. The following diagram illustrates a hierarchy of java design patterns.

\section{ABSTRACT FACTORY PATTERN}

A modest factory pattern always returns an occurrence of several probable classes dependent on this data to provide for it. However, an abstract-factory pattern technique is one range of generalization than this factory pattern technique. This pattern(s) can be recycled to reuse one of the multiple different related objects or classes each of which can return multiple different object(s) on request call. This pattern can be understood by the following garden maker factory.

\section{ABSTRACT FACTORY PATTERN TO CRYSTALLOGRAPHY-FERROIC POINT GROUPS}

Each geometrical-body or molecular arrangement is categorized by a group of symmetric operation(s) which when totally enumerated constitutes a point-group. There are seven well known crystal systems and all the lattices should fall under one or other of these categories. In turn these seven systems generate 32-point groups. The occurrence of physical property(s) can be determined by the symmetry operations of the group relating to a crystal. The abstract factory pattern method has been used to determine whether a crystal exhibits a physical property or not. Here the generating elements of a group are considered and the tensorial property is applied on them to determine the physical property(s) exhibited by the crystal. The basic structure and features of the main program consist the following

- In the place of the class plant of the garden application my object class is introduced.

- In the place of the garden class Abstract Factory class has been introduced.

- In the place of Gardener class Abstract Factory Application class is introduced to control the whole program.

1.

- By passing the parameter to the make element

2. ( ) of the Abstract Factory class 7 possible classes may be generated. But in the present context a single class which preserves all the property(s) is constructed.
- The present application consists of four server classes and one client class.

- Implement the operation(s) to carry the tasks and collaboration(s) in all the pattern(s).

Depending upon the purposes of the design pattern can be classified into three categories.

- Creational patterns: The objects created instead of initiating them directly. Here programs gain additional flexibility in determining which object(s) need to be produced for any given scenario.

- Structured patterns: These pattern(s) help in composing group(s) of objects into bigger structure(s) such as complex interface \& accounting info.

- Behavioral pattern: These patterns helps to describe communications between objects in the system \& the movement of control in a complicated program.

There are 23 design pattern(s) that were implemented in java in literature for different varieties of problems. The following diagram illustrates a hierarchy of java design patterns.

\section{RESULTS AND DISCUSSIONS}

The drop-down box of the java frames has been used to show the physical property(s) as well as the structure of all the 7 hexagonal point groups. The structure and the physical property(s) exhibited by the crystal for the point groups $6, \overline{6}, 6 / \mathrm{m}, 6 \mathrm{~mm}, 622, \overline{6} 2 \mathrm{~m}, 6 / \mathrm{mmm}$ is obtained by selecting each point group in the drop-down box of the application frame.

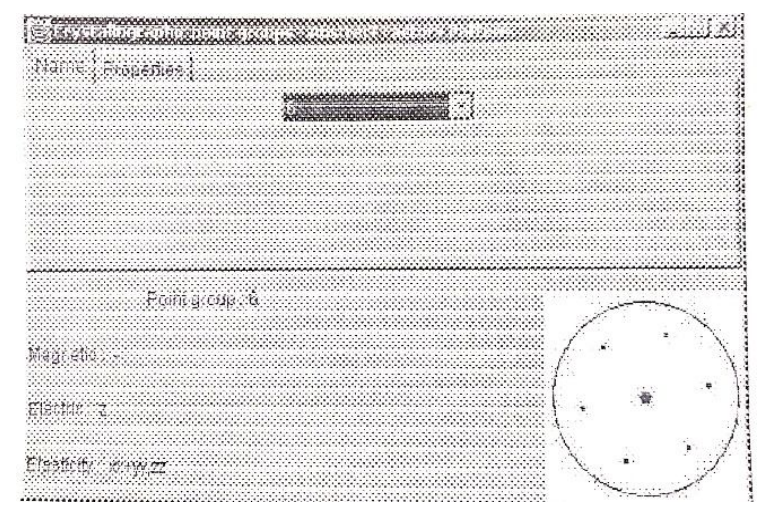

Figure 1: Stereographic projection and physical property(s) of hexagonal point group 6 


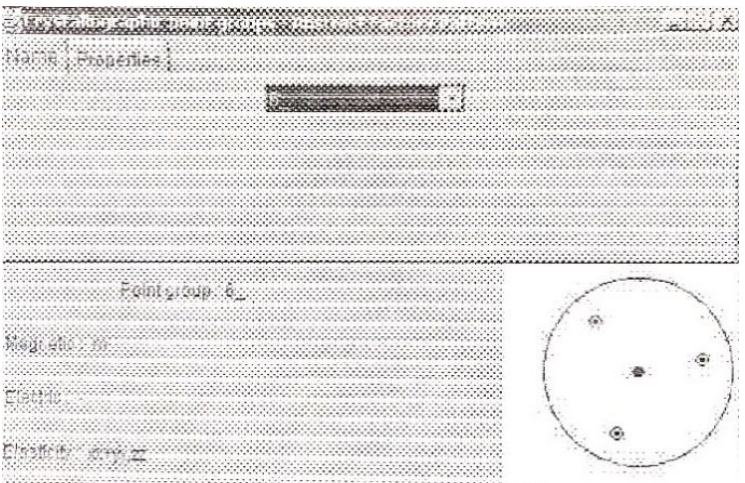

Figure 2 : Stereographic projection and physical property(s) of hexagonal point group $\overline{6}$

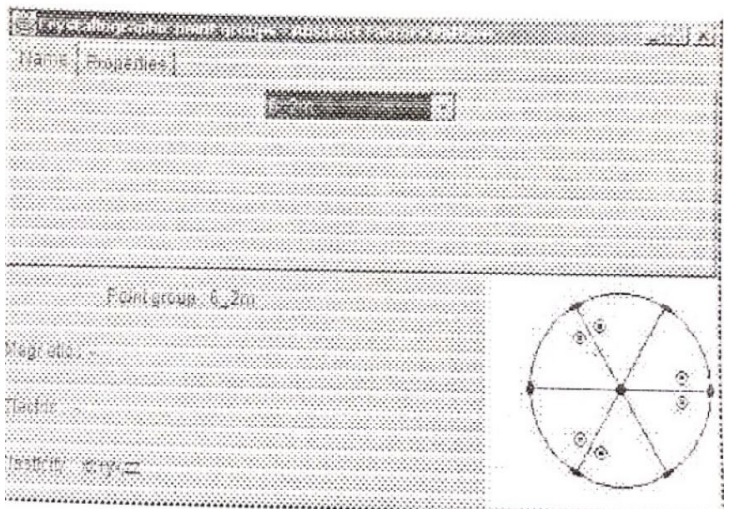

Figure 3: Stereographic projection and physical property(s) of hexagonal point group $\overline{6} 2 \mathrm{~m}$

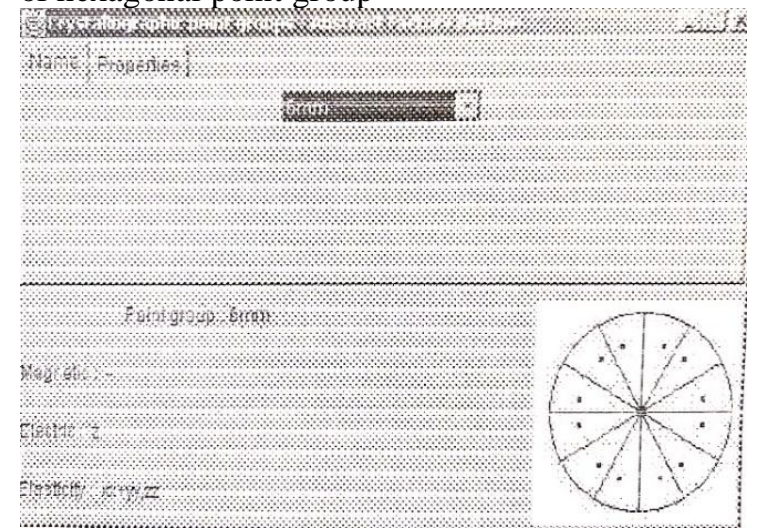

Figure 4: Stereographic projection and physical property(s) of hexagonal point group $6 \mathrm{~mm}$

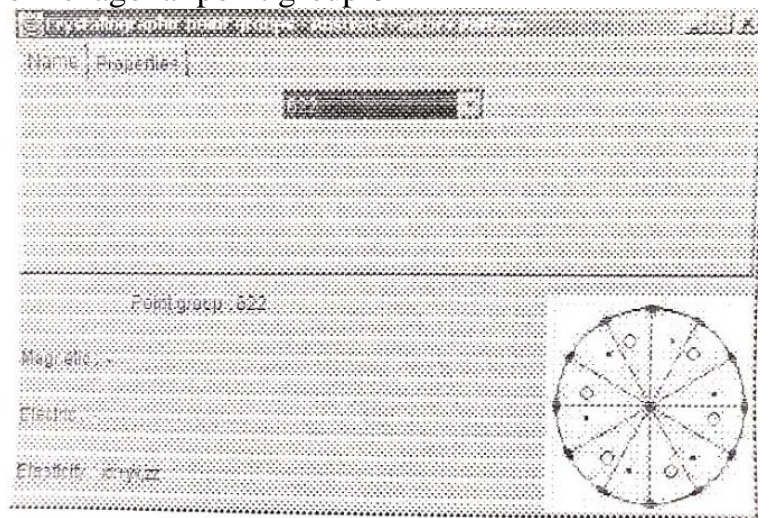

Figure 5: Stereographic projection and physical property(s) of hexagonal point group 622

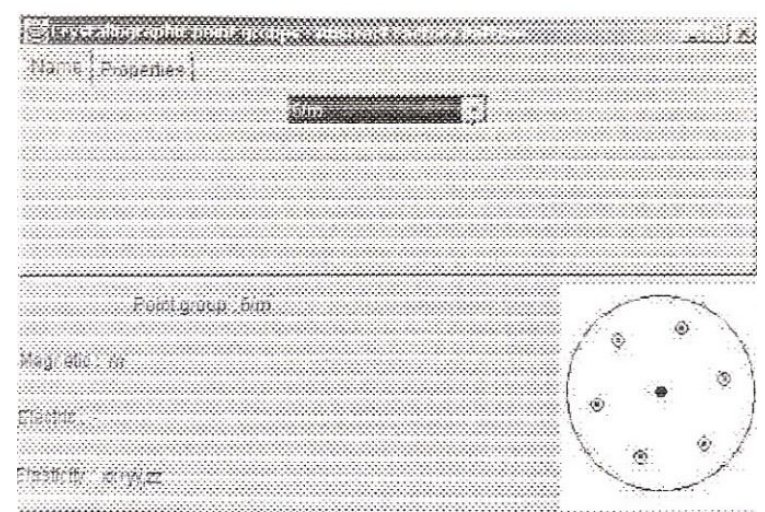

Figure 6: Stereographic projection and physical property(s) of hexagonal point group $6 / \mathrm{m}$

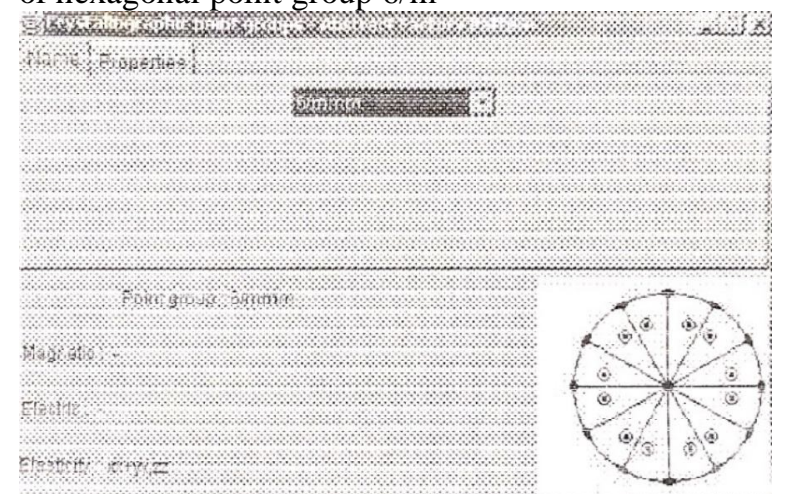

Figure 7: Stereographic projection and physical property(s) of hexagonal point group $6 / \mathrm{mm}$

\section{CONCLUSION}

In the method make element () of Abstract Factory class, based on the parameters passed, a new element is constructed which may take 7 possible forms, which returns the required outputs. This process eliminates a high degree of redundant code and execution time. Thus the basic object oriented features of the java design patterns i.e. the data abstraction and encapsulation etc. helps in better design for complex broadly generalized system. This signifies that the present work can also be extended to the other classes of 32 point groups and 212 non-magnetic ferroic species for their full or partial physical property(s) as well as their Stereographic-Projections of the geometrical bodies.

\section{REFERENCES}

1. Pree et al. Design patterns of object oriented software development Reading, Mass.: Addison-wesley, 1995

2. Aizu Keitsiro, Possible species of ferromagnetc, ferroelectric and ferroelastic crystal, Phys. Rev. B, 2, 754-772,1970.

3. Nagesh, P., Radha Madhavi, M., Surendra Prasad Babu, M. Reusable object oriented design for stereographic projections of tetragonal point groups, International Journal of Innovative 
Technology and Exploring Engineering, Volume.8,No.8,177-180,2019.

4. Lanka, S., Madhavi.M.R., Abusahmin, B. S., Puvvada, N., \& Lakshminarayana, V., Predictive data mining techniques for management of high dimensional big-data, Journal of Industrial Pollution Control, 33, 1430-1436,2017.

5. Ramesh Babu, A., Radha Madhavi, M., Sarala, S., \& Lakshminarayana, V., Tensor pairs and domain pairs of cesium dihydrogen phosphate using group theoretical techniques. Journal of Industrial Pollution Control, 33, 1475-1480,2017.

6. Madhavi, R., Karri, R. R., Sankar, D. S., Nagesh, P., \& Lakshminarayana, V., Nature inspired techniques to solve complex engineering problems. Journal of Industrial Pollution Control, 33(1), 1304-1311,2017.

7. Puvvada, N., \& Prasad Babu, M. S. Semantic web based banana expert system. International Journal of Mechanical and Production Engineering Research and Development, 8(3), 364-371,2018.

8. Venkata Ramana, N., Nagesh, P., Lanka, S., \& Karri, R. R. , Big data analytics and iot gadgets for tech savvy cities. doi:10.1007/978-3-030-03302-6_12,2019.

9. Venkata Ramana, N., Seravana Kumar, P. V. M., \& Nagesh, P., Analytic architecture to overcome real time traffic control as an intelligent transportation system using big data. International Journal of Engineering and Technology(UAE), 7(2.18 Special Issue 18), 7-11,2018.

10. Venkata Ramana, N., Nagesh, P., Seravana Kumar, P. V. M., \& Vignesh, U. IoT based scientific design to conquer constant movement control as a canny transportation framework utilizing huge information available in cloud networks. Journal of Advanced Research in Dynamical and Control Systems, 10(7 Special Issue), 1395-1402,2018.

11. Rajesh, B., Nagesh, P., Gowtham, K., Vivek, G., \& Srinivasu, N. A new scheme to safeguard data for cloud integrated internet things, International Journal of Innovative Technology and Exploring Engineering, 8(4), 174-178,2019.

12. Venkata Ramana, N., Kolli, C. S., Ravi Kumar, T., \& Nagesh, P. . Hybrid K-mir algorithm to predict type of lung cancer among stoicism. International Journal of Innovative Technology and Exploring Engineering, 8(4), 283-287,2019.

13. G Siva Nageswara Rao.et al. Efficient PIMRR Algorithm for Improving scheduling Criteria's in Real Time Systems, International Journal of Engineering and Technology (ISSN 2227-524X) volume 7, No. 2.32, Special Issue 32, pp 275-278, July. 2018.

14. G Siva Nageswara Rao.et al. Modern Vehicle Tracking and Monitoring System using Embedded Technology, International Journal of Recent
Technology and Engineering (IJRTE) (ISSN 2277-3878) volume 8, Issue 1, pp 1849-1851, May 2019.

15. G Siva Nageswara Rao.et al. Food Waste Protein sequence analysis using clustering and classification techniques, International Journal of Advanced tends in computer science and Engineering (ISSN 2278-3091) volume 8, Issue 5, pp 2289-2298, October 2019.

16. Vijaya, N., Madhavi, M. R.,et al., Boundary layer of a mixed convective nanofluid flowing over a vertical circular cylinder under the influence of magnetic field, heat radiation and outside surface temperature. International Journal of Mechanical and Production Engineering Research and Development, 8, pp. 411-420, 2018.

17. Gobinath Ravindra, M. Radha Madhavi Optimization of Zinc(II) Adsorption Using Agricultural Waste, International Journal of Engineering \& Technology, 7 (3.34) ,300-304,2018 